\title{
Motivationsinterventionen - Lernen aus erwartungswidrigen Befunden oder warum immer etwas rauskommt: Einführung in den Thementeil
}

\author{
Birgit Spinath
}

(C) Die Herausgeber und Springer Fachmedien Wiesbaden GmbH, ein Teil von Springer Nature 2019

Motivation ist eine zentrale Voraussetzung für Lernen und Leistung, die insbesondere aufgrund ihrer potentiellen Beinflussbarkeit durch gezielte Förderung für Forschung und Praxis in gleicher Weise relevant ist. In der Pädagogischen Psychologie sind daher zahlreiche Ansätze zur Förderung von Lern- und Leistungsmotivation entwickelt worden. Während sich die Forschung zur Wirksamkeit von Motivationsinterventionen in Lern- und Leistungskontexten längere Zeit in einer Phase niedriger Forschungsaktivität und geringer Innovation befand (vgl. z. B. Lazowski und Hulleman 2016; Rosenzweig und Wigfield 2016), erlebt sie seit rund 10 Jahren eine Renaissance. In einigen der renommiertesten wissenschaftlichen Zeitschriften wie Science und Proceedings of the National Academy of Sciences werden Befunde aus Interventionsstudien berichtet (z. B. Hulleman und Harackiewicz 2009; Rozek et al. 2017), die mit zum Teil erstaunlich geringem Aufwand verblüffende Ergebnisse erzielen (für einen Überblick über die theoretischen und methodischen Ansätze siehe Yeager und Walton 2011). Diese Publikationen lösten in der Motivationsforschung rege Betriebsamkeit aus, denn zahlreiche Wissenschaftlerinnen und Wissenschaftler rezipieren die Befunde und versuchen sie zu replizieren. Einige dieser Versuche gelingen, andere nicht. Letzteres dringt häufig nicht an die Öffentlichkeit, sondern die Studien verschwinden in der Regel in den Schubladen (File-Drawer-Effekt, Publication Bias), weil sich Null-Befunde bekanntlich kaum publizieren lassen, wenn sie nicht präregistriert wurden oder aus Multi-Lab-Studien hervorgehen, die häufig genau mit dem Ziel der Entmystifizierung eines Befundes initiiert werden. Im persönlichen Gespräch heißt es häufig, es sei „nichts rausgekommen“, und schnell wird die nächste Studie begonnen. Diese Praktiken binden eine große Menge an Ressourcen, die besser eingesetzt wären, wenn die Wissenschaftsgemeinde auch aus den

B. Spinath $(\bowtie)$

Universität Heidelberg, Hauptstr. 47-51, 69117 Heidelberg, Deutschland

E-Mail: birgit.spinath@psychologie.uni-heidelberg.de 
Null-Befunden lernen könnte. Auch trägt diese Praxis zum Problem der falsch positiven Befunde bei (Simmons et al. 2011), indem die erwartungswidrigen Befunde als Gegengewicht zu den publizierten positiven Befunden fehlen. Das Hauptanliegen des vorliegenden Thementeils ist es, von dieser gängigen Praxis abzuweichen, um aufzuzeigen, dass erwartungswidrige Befunde lehrreich sein können.

Im Folgenden werden Studien vorgestellt, die Null-Befunde oder aber andere erwartungswidrige Ergebnisse zu Tage gefördert haben. Folgende Einschlusskriterien wurden angelegt: Es handelt sich um Interventionsstudien in Lern- und Leistungskontexten, bei denen Motivation die Haupt Zielvariable ist. Die Studien haben in Hinblick auf eine gut etablierte Theorie oder einen gut etablierten Befund nichterwartungskonforme Ergebnisse hervorgebracht. Aus diesen nicht-erwartungskonformen Befunden muss für andere Wissenschaftlerinnen und Wissenschaftler ein Lerneffekt entstehen, der in den Studien herausgearbeitet wird. Hierfür nicht geeignet sind Studien, bei denen zwar nachvollziehbar ist, warum die erwarteten Effekte nicht aufgetreten sind, wobei diese Gründe jedoch im Vorfeld der Studien hätten bekannt sein können (z. B. zu geringe Stichprobengröße, ungeeignete Stichprobe, Deckeneffekte in der abhängigen Variablen, geringe psychometrische Güte der Messinstrumente). Interventionsstudien erfordern stets Vorstudien, in denen eine Bedarfsermittlung erfolgt, Messinstrumente erprobt und die praktische Umsetzbarkeit der Intervention überprüft werden.

Alle vier in diesem Thementeil vorgestellten Studien sind experimentelle Studien, in denen die Wirkung von Motivationsinterventionen in Lern- und Leistungskontexten in Kontrollgruppen-Designs untersucht wurden. Bei jeder Studie zeigten sich erwartungswidrige Befunde, die von den Autorenteams hinsichtlich forschungsmethodischer, theoretischer, kultureller und weiterer Erklärungen diskutiert werden.

Die Studie von Heide Piesch, Isabelle Häfner, Hanna Gaspard, Barbara Flunger, Benjamin Nagengast and Judith Harackiewicz (2019) ist eine auf deutsche Verhältnisse übertragene, konzeptuelle Replikation einer Nützlichkeitsintervention, die sich in den USA in vorauslaufenden Studien als wirksam erwiesen hatte (z. B. Harackiewicz et al. 2012). Mittels einer Website wurden Eltern Informationen über die Nützlichkeit bestimmter schulischer Inhalte für verschiedene Berufsfelder zur Verfügung gestellt, und die Eltern wurden angeregt, mit ihren Kindern über diese Informationen zu sprechen. Kontraintuitiver Weise zeigte die Intervention negative Effekt auf die elterliche Unterstützung bei der Berufswahl und die Bewertung deren Wichtigkeit solcher Unterstützung. Darüber hinaus zeigten sich keine signifikanten Unterschiede zwischen den Bedingungen.

Marc Philipp Janson und Oliver Dickhäuser (2019) berichten über eine Studie, in der die Wirkung von Wenn-Dann-Plänen (Gollwitzer 1999) in einer Prüfungsphase im Studium untersucht wurde. Die Studierenden waren darin angeleitet worden, ihre Prüfungsvorbereitungen entweder durch Wenn-Dann-Pläne oder durch mentales Konstrastieren (Oettingen et al. 2000) zu unterstützen. Zwar zeigte sich wie erwartet, dass Studierende, die in Wenn-Dann-Plänen unterwiesen worden waren, in der Prüfung besser abschnitten, jedoch wurden diese Effekte nicht wie erwartet über einen früheren Lernbeginn und größere Lernhäufigkeit vermittelt. Damit fehlt jedoch ein wesentlicher Baustein in der Erklärung der Befunde. 
Der Beitrag von Katharina Benning, Martin Daumiller, Anna K. Praetorius, Gerlinde Lenske, Oliver Dickhäuser und Markus Dresel (2019) berichtet über eine Intervention für Lehrkräfte zur Verbesserung von Zielorientierungen und motivationsförderlichem Unterrichtshandeln. In Form eines Workshops wurde Lehrkräften an drei Tagen Wissen zu Zielorientierungen vermittelt und dessen praktische Anwendung geübt. Obwohl sich seitens der Lehrkräfte ein hoher subjektiver Lernertrag, wahrgenommener Nutzen sowie große Zufriedenheit mit dem Interventionsansatz zeigten, ergaben sich im Vergleich zur Kontrollgruppe keine Effekte auf Lehrkraftziele, wahrgenommene Zielorientierung in der Klasse oder die Motivation der Schülerinnen und Schüler.

Nora Maria Foerst, Andreas Pfaffel, Julia Krug, Christiane Spiel und Barbara Schober (2019) erprobten eine App, deren Ziel es ist, die selbstregulatorischen und motivationalen Kompetenzen von Studierenden zu steigern, speziell in der Phase des Schreibens ihrer Bachelorarbeit. Entgegen den Zielen der Intervention zeigten sich sowohl in der Treatment- als auch den Kontrollgruppen signifikante negative Veränderungen in der Einschätzung des eigenen Wissens über Selbstregulationsstrategien sowie in den Attributionsmustern für Erfolg bzw. Misserfolg.

Welche Erklärungen die Autorenteams für ihre Ergebnisse gefunden haben, erfahren Sie bei der Lektüre der Originalarbeiten. Wie ich finde, handelt es sich bei allen Studien um lesenswerte Beispiele dafür, dass erwartungswidrige Effekte lehrreich sein können. In diesem Sinne möchte ich appellieren, dass wir erwartungswidrige Befunde ernst nehmen und interpretieren, denn bei sorgfältig entwickelten Studien kommt immer etwas heraus und es gibt immer etwas daraus zu lernen.

\section{Literatur}

Benning, K., Daumiller, M., Praetorius, A. K., Lenske, G., Dickhäuser, O., \& Dresel, M. (2019). Evaluation eines Interventionsansatzes zur Verbesserung von Motivation und motivationsförderlichem Unterrichtshandeln von Lehrkräften auf Basis der Zielorientierungstheorie. Unterrichtswissenschaft. https://doi.org/10.1007/s42010-018-0025-9.

Foerst, N. M., Pfaffel, A., Krug, J., Spiel, C., \& Schober, B. (2019). Self-Regulated Learning (SRL) in der Tasche? - Eine SRL-Interventionsstudie im App-Format. Unterrichtswissenschaft. https://doi.org/10. 1007/s42010-019-00046-7.

Gollwitzer, P. M. (1999). Implementation intentions: Strong effects of simple plans. American Psychologist, 54, 493-503.

Harackiewicz, J. M., Rozek, C. S., Hulleman, C. S., \& Hyde, J. S. (2012). Helping parents to motivate adolescents in mathematics and science: An experimental test of a utility-value intervention. Psychological Science, 23, 899-906.

Hulleman, C.S., \& Harackiewicz, J. M. (2009). Promoting interest and performance in high school science classes. Science, 326, 1410-1412.

Janson, M.P., \& Dickhäuser, O. (2019). Prüfungsvorbereitung an der Hochschule mittels Lernsoftware: Die Effekte von Wenn-Dann-Plänen. Unterrichtswissenschaft. https://doi.org/10.1007/s42010-0180018-8.

Lazowski, R. A., \& Hulleman, C.S. (2016). Motivation interventions in education: A metaanalytic review. Review of Educational Research, 86, 602-640.

Oettingen, G., Hönig, G., \& Gollwitzer, P. M. (2000). Effective self-regulation of goal attainment. International Journal of Educational Research, 33, 705-732.

Piesch, H., Häfner, I., Gaspard, H., Flunger, B., Nagengast, B., \& Harackiewicz, J. M. (2019). Helping parents support adolescents' career orientation: Effects of a parent-based relevance intervention. $U n$ terrichtswissenschaft. https://doi.org/10.1007/s42010-018-0024-x. 
Rosenzweig, E.Q., \& Wigfield, A. (2016). STEM motivation interventions for adolescents: A promising start, but further to go. Educational Psychologist, 51, 146-163.

Rozek, C.S., Svoboda, R.C., Harackiewicz, J.M., Hulleman, C.S., \& Hyde, J.S. (2017). Utility-value intervention with parents increases students' STEM preparation and career pursuit. Proceedings of the National Academy of Sciences of the United States of America, 114, 909-914.

Simmons, J.P., Nelson, L. D., \& Simonsohn, U. (2011). False-positive psychology: Undisclosed flexibility in data collection and analysis allows presenting anything as significant. Psychological Science, 22, 1359-1366.

Yeager, D. S. \& Walton, G. M. (2011). Social-psychological interventions in education: they're not magic. Review of Educational Research, 81, 267-301. 